\title{
Comunidades y gremios de parasitoides (Hymenoptera: Ichneumonidae) en cultivo de maíz y selva baja caducifolia circundante
}

\section{Parasitoids assemblages and guilds (Hymenoptera: Ichneumonidae) on maize and adjacent dryforest}

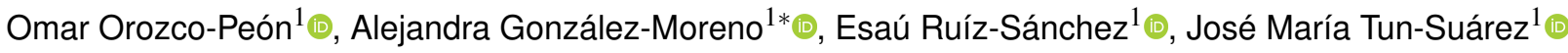 \\ ${ }^{1}$ Tecnológico Nacional de México/ I.T. Conkal. Av. Tecnológico S/N. CP. 97345. Conkal, Yucatán, México. \\ *Autor de correspondencia: alejandra.gonzalez@itconkal.edu.mx
}

Artículo científico recibido: 31 de agosto de 2018 aceptado: 28 de enero de 2019

RESUMEN. En áreas tropicales se conoce poco sobre la diversidad de parasitoides, especialmente en cultivos; donde se ha demostrado que estos insectos son sensibles al aumento de la superficie agrícola. El objetivo del trabajo fue evaluar la diversidad y composición de especies de parasitoides en cultivos de maíz de diferente superficie con selva baja caducifolia circundante. Se colocaron seis trampas Malaise: dos en un cultivo de maíz de 10 ha, dos en maíz de 50 ha y dos en selva, que funcionaron durante el ciclo fenológico del maíz, en las que se realizaron colectas semanales. Se recolectaron 1149 individuos, que representan 43 géneros de los que 21 son nuevos registros para el estado de Campeche, México. No se encontraron diferencias en el número de géneros presentes en los sitios y la composición de comunidades fue similar, lo que refleja la migración de géneros entre la selva y los agroecosistemas; pero si presentaron diferencias en los géneros dominantes, lo que sugiere que los parasitoides responden al hábitat. Se encontraron siete gremios de parasitoides, dominando los parasitoides que atacan fitófagos ocultos. La diversidad de géneros fue similar entre la selva y maíz de 50 ha, la diversidad de gremios se relaciona con el tamaño del hábitat, áreas de mayor tamaño, tienen mayor biodiversidad. Los géneros que dominaron las comunidades fueron Eiphosoma, Pristomerus, Microcharops y Casinaria, los cuales, con excepción del último, han sido registrados como controladores biológicos de Spodoptera frugiperda en maíz.

Palabras clave: Campeche, diversidad, enemigos naturales, nuevos registros, Zea mays.

ABSTRACT. In tropical areas little is known about the diversity of parasitoids, especially in cropped areas; which have shown that these insects are particularly sensitive to the increase in agricultural activities. The aim was to evaluate the diversity and composition of parasitoid species in maize fields with different area in a matrix of low deciduous forest. Six Malaise traps were placed: two in a maize field of 10 ha, two in maize field of 50 ha and two into dryforest, which were functioning during the maize phaenological cycle (April-November 2016) with weekly collections. 1149 individuals were found represented in 43 genera, which 21 as new records for the Campeche State, Mexico. No differences were found in the number of genera present among sites, and the composition of the communities were very similar to each other, which reflects the migration of genera between the forest and maize fields. But differences in the dominant genera were founded, suggesting that the parasitoids respond to particularities of the habitat. Seven guilds of parasitoids were found, dominating the parasitoids that attack hidden phytophages. The diversity of genera was similar between forest and 50 ha maize field, and diversity of guilds was related with the size of the habitat, larger areas will have higher biodiversity. The genera that dominated the communities were Eiphosoma, Pristomerus, Microcharops and Casinaria, which, with the exception of the latter, have been registered as biological controllers of Spodoptera frugiperda in maize.

Key words: Campeche, diversity, natural enemies, new records, Zea mays.

\section{INTRODUCCIÓN}

El maíz es un cultivo de gran importancia económica, social y cultural para México, con una superficie cosechada de 5039934 ha (SIAP 2018). Al igual que muchos cultivos es atacado por diferentes 
plagas (Valdez-Torres 2012), de las cuales una de las más graves es el gusano cogollero Spodoptera frugiperda (J.E. Smith) (Lepidoptera: Noctuidae) (Nuessly et al. 2007). Este fitófago se controla con insecticidas, pero debido a la creciente necesidad de una agricultura sostenible, el control biológico con parasitoides, ha adquirido importancia (Maciel y Araiza 2015).

Los parasitoides son insectos principalmente del orden Hymenoptera (abejas, avispas y hormigas), que en su etapa larval se desarrollan y alimentan dentro o sobre otros artrópodos, a los que matan al final del proceso (Godfray 1994). Estos insectos mantienen el balance natural de los ecosistemas al ser reguladores de insectos fitófagos, por lo que se utilizan en el control biológico de plagas, lo que les confiere valor económico. En términos de diversidad son relevantes por su gran número de especies, por lo que son idóneos como modelos en estudios de biodiversidad (Veijalainen et al. 2013). Existen varios estudios de parasitoides en maíz que describen la relación hospedero-parasitoide (García-Gutierrez et al. 2013, González-Maldonado et al. 2014, Maciel y Araiza 2015, Gutiérrez-Ramírez et al. 2015) y otros se enfocan a la diversidad de parasitoides en los agroecosistemas (Hay-Roe et al. 2016, Meagher et al. 2016, Coronado-Blanco et al. 2017, Rojas et al. 2017). En la región sureste del país, se tienen algunos trabajos sobre diversidad de parasitoides del Estado de Yucatán (Ruíz-Cancino et al. 2014, González-Moreno et al. 2015, 2018), realizados principalmente en Áreas Naturales Protegidas, además del trabajo sobre especies de Ichneumonidae en Escárcega, Campeche (García-Ramírez et al. 2016). Específicamente, sobre parasitoides de maíz en el Estado de Yucatán, se han registrado cuatro especies de parasitoides emergidos de $S$. frugiperda (Delfín-González et al. 2007), mientras que ChayHernández et al. (2016) estudiaron la importancia de la vegetación circundante al cultivo de maíz asociado con frijol.

Los estudios de diversidad de parasitoides indican que son sensibles al aumento de la superficie agrícola, debido a su tamaño, baja capacidad de dispersión, alta especificidad de hospederos y su dependencia al hábitat que les proporciona el hospedero y los recursos florales (Inclán et al. 2014). En general, la mayoría de los estudios concluyen que la diversidad del paisaje favorece la diversidad de parasitoides, especialmente cuando los paisajes están conformados por cultivos rodeados de vegetación natural (Landis et al. 2000). Pero la manera en que este aumento en superficie de cultivo impacta sobre la composición de especies de parasitoides no está claro (Inclan et al. 2014). Por lo que el objetivo del presente estudio fue conocer la diversidad y composición de comunidades de parasitoides en cultivos de maíz de diferente tamaño en una matriz de selva baja caducifolia.

\section{MATERIALES Y MÉTODOS}

El estudio se realizó en la localidad de Becal, Campeche, México, región productora de maíz. Se escogieron tres sitios de muestreo, dos unidades de producción de maíz, la primera de 10 ha y la segunda de 50 ha, mientras que el tercer sitio fue la selva baja caducifolia circundante de los cultivos. Ambos agroecosistemas tuvieron el mismo manejo agronómico. La preparación del terreno se realizó por medio de dos pasos de rastra, para luego sembrar el híbrido de maíz DK 7500 amarillo. El control de malezas preemergentes se realizó con el herbicida Faena Fuerte, Defensa y Harnes Extra en dosis de 2, 1 y $2 \mathrm{~L} \mathrm{ha}^{-1}$, una vez que emergieron las plantas de maíz se realizó la aplicación postemergente de Paraquat a dosis de $2 \mathrm{~L} \mathrm{ha}^{-1}$ con Amina + Picloram a $1 \mathrm{~L} \mathrm{ha}^{-1}$.

La dosis de fertilización por hectárea fue de $110 \mathrm{~kg}$ de N, $46 \mathrm{~kg}$ de P y $30 \mathrm{~kg}$ de K, que se realizó por medio de dos aplicaciones de forma manual. La primera aplicación (18 kg N, 46 kg de P y $30 \mathrm{~kg}$ de K) se realizó al momento de la siembra con Fosfato Diamonico y Cloruro de Potasio. La segunda aplicación (92 kg de N) se realizó a los 40 días después de la siembra con Urea. Para el control químico del gusano cogollero ( $S$. frugiperda) se aplicó el insecticida Rimon $10 \mathrm{EC}$ a dosis de $100 \mathrm{ml} \mathrm{ha}^{-1}$ y Látigo $\mathrm{L}$ a dosis de $1 \mathrm{~L} \mathrm{ha}^{-1}$.

En cada sitio se colocaron dos trampas 
Malaise que funcionaron durante todo el ciclo del maíz de abril a noviembre de 2016. Las muestras se tomaron cada semana y se procesaron de acuerdo con las técnicas curatoriales convencionales en el laboratorio de plagas agrícolas del Instituto Tecnológico de Conkal, Yucatán, México. La identificación de los ejemplares de Ichneumonidae, a nivel de género se realizó con las claves taxonómicas de Gauld (1991, 1997, 2000 y 2002).

El análisis de datos se realizó describiendo las comunidades en términos de riqueza de géneros, composición de las comunidades y diversidad. Para determinar si el inventario de los géneros estaba completo se construyeron curvas de acumulación mediante el programa EstimateS 9.1.0 (Colwell 2016), con el estimador de cobertura basado en la incidencia (ICE) que se basa en los géneros encontradas en menos de 10 muestras, recomendado para este tipo de muestras de artrópodos. Para determinar si existían diferencias en las abundancias de parasitoides entre sitios se realizó un análisis no paramétrico Kruskal-Wallis, con una comparación multiple post-hoc Mann-Whitney con corrección de Bonferroni, para conocer que sitios eran diferentes entre sí. Para analizar la composición de las comunidades de Ichneumonidae se construyeron curvas de rango-abundancia o curvas de Whittaker, para analizar cómo se distribuye la riqueza y la abundancia de cada género dentro de cada sitio de muestreo. Se analizó la diversidad con el índice de dominancia de Simpson con el programa Species Diversity and Richness III (Magurran 2004). También se realizó un análisis de gremios tróficos considerando la clasificación de Garbarczyk y Sawoniewicz (1984) modificada por Mazón y Bordera (2014) sobre el grado de especialización y el tipo de hospedero que utilizan los géneros de los parasitoides encontrados: parasitoides de larvas fitófagas ocultas (PLFO), parasitoides de larvas fitófagas expuestas (PLFE), parasitoides de pupas (PP), parasitoides con biología desconocida (PBD), parasitoides de larvas xilófagas (PLX), parasitoides de larvas saprófagas (PLS), parasitoides de larvas que se alimentan de polen y nectar (PLAPN). Para lo cual también se describieron los ensambles de gremios mediante curvas de
Whittaker, así como el análisis de diversidad y análisis de correspondencia para determinar la relación entre gremios y los sitios estudiados, con el software PAST (Hammer et al. 2001).

\section{RESULTADOS}

\section{Riqueza, composición y diversidad de géneros}

Se recolectaron 1149 especímenes de la familia Ichneumonidae pertenecientes a 12 subfamilias y 43 géneros, incrementando la fauna conocida para el Estado de Campeche, con 21 nuevos registros (Tabla 1). Los géneros capturados en los tres sitios representan el 66,74 y $62 \%$ de los estimados según el índice ICE para el cultivo de maíz de 10 ha, cultivo de maíz de 50 ha, y la selva, respectivamente. Si consideramos los sitios por separado la tasa de adición de géneros fue similar en los dos sitios con cultivo de maíz, independientemente de la superficie (Figura 1). En la selva, la acumulación de especies fue mayor que en los sitios con cultivo de maíz, siendo donde faltó mayor esfuerzo de muestreo. En términos de riqueza de Ichneumonidae, no se encontraron diferencias en el número de géneros encontrados entre sitios, según los intervalos del confianza al 95\% (Figura 1).

Con relación a la abundancia se recolectó el mayor número de individuos en la selva, seguido del cultivo de maíz de 50 y 10 ha, con diferencias significativas entre la selva y el cultivo de maíz con 10 ha $(H=9.86, p<0.006)$. Estas diferencias también se encontraron en la composición de las comunidades, ya que en los sitios con cultivos de maíz se tuvieron comunidades menos equitativas que en la selva baja caducifolia. De acuerdo con la pendiente de las curvas de Whittaker, los géneros que dominan las comunidades entre los sitios son diferentes. En la selva el género dominante fue Casinaria, en el cultivo de maíz de 50 ha fue Microcharops y en 10 ha fue Pristomerus. Eiphosoma fue el segundo género más abundante en los tres sitios (Figura 2). En términos de diversidad, la selva caducifolia circundante y el cultivo de maíz de 50 ha tuvieron la misma diversidad de parasitoides, mientras que el sitio menos diverso fue el cultivo de maíz de 10 ha (Tabla 2). 
Orozco-Peón et al.

Tabla 1. Abundancia de subfamilias y géneros de Ichneumonidae en dos parcelas de maíz y selva baja caducifolia circundante.

\begin{tabular}{|c|c|c|c|c|}
\hline Subfamilias y Géneros & Maíz de 10 ha. & Maíz de 50 ha. & Selva baja & Total \\
\hline \multicolumn{5}{|l|}{ Anomaloninae } \\
\hline Anomalon & 5 & 2 & 1 & 8 \\
\hline Ophionellus* & 7 & 2 & 1 & 10 \\
\hline Podogaster* & 1 & 0 & 1 & 2 \\
\hline \multicolumn{5}{|l|}{ Banchinae } \\
\hline Diradops & 0 & 2 & 1 & 3 \\
\hline Meniscomorpha & 1 & 1 & 6 & 8 \\
\hline Mnioes* & 2 & 0 & 1 & 3 \\
\hline \multicolumn{5}{|l|}{ Brachycyrtinae } \\
\hline Brachycyrtus* & 0 & 10 & 1 & 11 \\
\hline \multicolumn{5}{|l|}{ Campopleginae } \\
\hline Campoplex* & 0 & 1 & 3 & 4 \\
\hline Casinaria & 4 & 1 & 213 & 218 \\
\hline Charops* & 4 & 5 & 2 & 11 \\
\hline Dusona* & 0 & 7 & 7 & 14 \\
\hline Microcharops* & 16 & 117 & 44 & 177 \\
\hline Venturia* & 0 & 1 & 8 & 9 \\
\hline \multicolumn{5}{|l|}{ Cremastinae } \\
\hline Eiphosoma & 57 & 112 & 152 & 321 \\
\hline Pristomerus & 92 & 60 & 3 & 155 \\
\hline Temelucha & 2 & 1 & 0 & 3 \\
\hline Trathala* & 0 & 1 & 0 & 1 \\
\hline Xiphosomella* & 2 & 0 & 9 & 11 \\
\hline \multicolumn{5}{|l|}{ Cryptinae } \\
\hline Acerastes & 1 & 1 & 7 & 9 \\
\hline Agonocryptus* & 1 & 0 & 40 & 41 \\
\hline Baltazaria & 1 & 0 & 0 & 1 \\
\hline Baryceros & 0 & 0 & 5 & 5 \\
\hline Camera* & 0 & 0 & 7 & 7 \\
\hline Cryptanura & 0 & 0 & 3 & 3 \\
\hline Diapetimorpha & 1 & 0 & 1 & 2 \\
\hline Endasys ${ }^{*}$ & 0 & 0 & 63 & 63 \\
\hline Lampocryptus* & 0 & 0 & 2 & 2 \\
\hline Lymeon & 0 & 0 & 1 & 1 \\
\hline Mallochia* & 4 & 0 & 1 & 5 \\
\hline Messatoporus* & 2 & 3 & 0 & 5 \\
\hline Polycyrtus* & 0 & 1 & 0 & 1 \\
\hline Xenarthron* & 0 & 0 & 1 & 1 \\
\hline Xylophorus ${ }^{\star}$ & 0 & 0 & 1 & 1 \\
\hline \multicolumn{5}{|l|}{ Ichneumoninae } \\
\hline Ichneumoninae sp 1 & 1 & 2 & 4 & 7 \\
\hline Ichneumoninae sp 2 & 0 & 2 & 1 & 3 \\
\hline Ichneumoninae sp 3 & 0 & 0 & 1 & 1 \\
\hline Ichneumoninae sp 4 & 0 & 0 & 4 & 4 \\
\hline \multicolumn{5}{|l|}{ Labeninae } \\
\hline Grotea* & 0 & 2 & 1 & 3 \\
\hline Labena & 1 & 4 & 1 & 6 \\
\hline \multicolumn{5}{|l|}{ Neorhacodinae } \\
\hline Neorhacodinae sp & 0 & 1 & 0 & 1 \\
\hline \multicolumn{5}{|l|}{ Ophioninae } \\
\hline Ophioninae sp & 0 & 2 & 2 & 4 \\
\hline \multicolumn{5}{|l|}{ Phrudinae } \\
\hline Phrudinae sp & 0 & 1 & 0 & 1 \\
\hline \multicolumn{5}{|l|}{ Pimplinae } \\
\hline Neotheronia & 1 & 0 & 2 & 3 \\
\hline Total & 206 & 342 & 601 & 1149 \\
\hline
\end{tabular}

${ }^{*}$ Nuevos registros para el Estado de Campeche. 


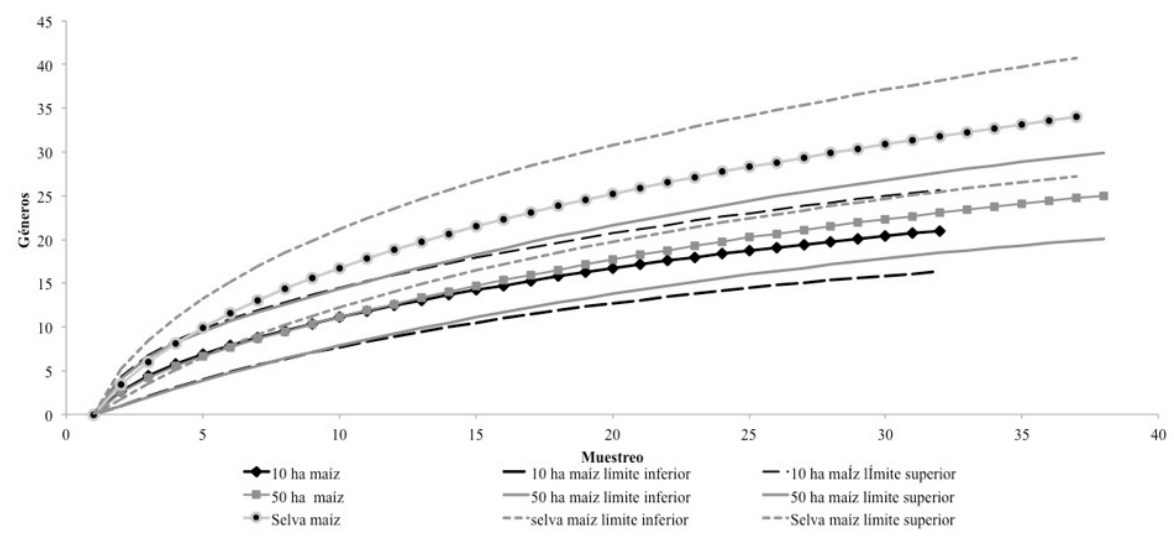

Figura 1. Curvas de acumulación de individuos de Ichneumonidae muestreados en dos sitios con cultivos de maíz de (10 y 50 ha) y selva baja caducifolia circundante de maíz.

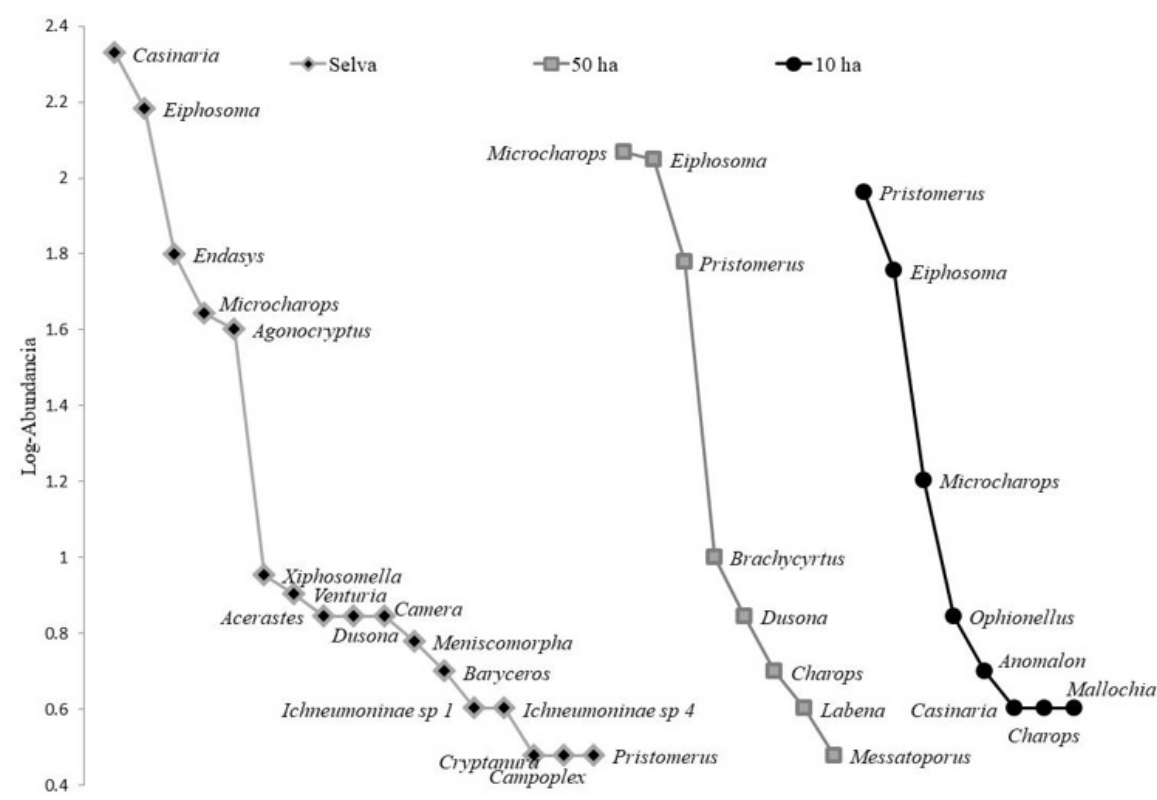

Figura 2. Curvas de Whittaker de géneros de Ichneumonidae en sitios con maíz de 10 y 50 ha y una selva baja caducifolia.

Tabla 2. Diversidad alfa de Simpson de géneros y gremios de Ichneumonidae en dos parcelas de maíz y selva baja caducifolia circundante.

\begin{tabular}{lcccccc}
\hline Sitios & $\begin{array}{c}\text { Índice } \\
\text { Géneros }\end{array}$ & $\begin{array}{c}\text { Límite } \\
\text { Inferior }\end{array}$ & $\begin{array}{c}\text { Límite } \\
\text { superior }\end{array}$ & $\begin{array}{c}\text { Índice } \\
\text { de Gremios }\end{array}$ & $\begin{array}{c}\text { Límite } \\
\text { inferior }\end{array}$ & $\begin{array}{c}\text { Límite } \\
\text { Superior }\end{array}$ \\
\hline Selva & 4.76 & 4.26 & 5.25 & 2.94 & 2.77 & 3.09 \\
Maíz 50 ha & 3.92 & 3.54 & 4.33 & 2.34 & 2.17 & 2.51 \\
Maíz 10 ha & 3.55 & 2.98 & 4.11 & 1.60 & 1.42 & 1.83 \\
\hline
\end{tabular}

Bootstrap con $95 \%$ de confiabilidad.

\section{Gremios tróficos}

Se encontraron siete gremios de parasitoides, los cuales fueron PLFO (512 individuos,
8 géneros), PLFE (438 individuos, 8 géneros), PP (143 individuos, 12 géneros), PBD (35 individuos, 11 géneros), PLX (9 individuos, 2 géneros), PLS (8 indi- 
viduos, 1 género) y PLAPN (5 individuos, 1 género). Las comunidades conformadas por los gremios de parasitoides también fueron diferentes en cada uno de los sitios, en el cultivo de maíz de 10 ha, el gremio dominante fue PLFO; en el cultivo de maíz de 50 ha dominaron los gremios de parasitoides PLFO y PLFE; mientras que en la selva dominaron los gremios PLFO, PLFE y PP (Figura 3). Con relación a la diversidad, los tres sitios presentan diferencias, siendo la selva el sitio más diverso, seguido del cultivo de maíz de 50 ha y por último el cultivo de maíz de 10 ha, mostrando una relación entre gremios y hábitats, en la selva se encontró una asociación más cercana entre PLFE y PP y en el maíz los parasitoides PLFO y PLAPN (Figura 4).

\section{DISCUSIÓN}

Los resultados obtenidos aumentan el conocimiento de Ichneumonidae a un total de 17 subfamilias y 49 géneros para el estado de Campeche, lo que representa $13 \%$ de los géneros registrados para el país (Ruíz-Cancino et al. 2014). Esto refleja que la biodiversidad de parasitoides es desconocida, ya que los resportes previos indicaban 14 subfamilias, 28 géneros y 11 especies (García-Ramírez et al. 2016). El incremento de la riqueza genérica, refleja la necesidad de realizar colectas en diferentes tipos de sistemas, tanto naturales como manejados, ya que con muestreos de un solo cultivo en un corto tiempo incrementó la biodiversidad en un $71 \%$.

La pendiente de las curvas de acumulación sugiere un buen esfuerzo de muestreo con dos trampas Malaise abarcando todo el ciclo del cultivo del maíz. Los resultados sobre la riqueza de géneros encontrada es similar a los encontrados en otro agroecosistema de maíz, donde se reportan 40 géneros (Chay-Hernández et al. 2006). El no encontrar diferencias en la riqueza, sugiere que tanto el agroecosistema de maíz, independientemente de su tamaño, como la selva alberga el mismo número de géneros de parasitoides de la familia Ichneumonidae. Esto puede deberse a que las áreas agrícolas rodeadas de sistemas naturales favorecen la migración de especies entre ambos sistemas, debido a la proximidad de los sitios, obteniendo recursos alternativos, como refugios, hospederos y comida (Landis et al. 2000, Hochberg e Ives 2000), lo que refuerza la importancia de mantener remanentes de vegetación dentro de los cultivos agrícolas.

Las diferencias en la abundancia de parasitoides de la selva y cultivo de maíz de 10 ha, puede explicarse con la biogeografia de islas, ya que áreas grandes tienen mayor probabilidad de alojar mayor biodiversidad (MacArthur y Wilson 1967). Con relación a la composición de las comunidades, es de esperarse que se conformaran de géneros diferentes entre la selva y los cultivos de maíz, pero que los géneros presentes entre lo cultivos de maíz, fueran más similares entre sí, en comparación con la selva. Esto puede deberse a que en los agroecosistemas únicamente se encontraba la especie $Z$. mays, por lo que los volátiles emitidos por esta planta estarían atrayendo las mismas especies de parasitoides, a diferencia de los múltiples volátiles que se pueden emitir por las diferentes especies vegetales de la selva (Godfray 1994), lo que atrae mayor riqueza de parasitoides, ya que volátiles producidos por insectos de diferentes especies de plantas, atraen determinadas especies de parasitoides (Godfray 1994).

Pese a las similitudes encontradas en los géneros que conformaron las comunidades, los géneros dominantes fueron diferentes, incluso en ambos sitios de cultivo de maíz. Se ha registrado que un mismo parasitoide puede tener diferente comportamiento, incluso en la misma planta hospedera, si cambia alguna característica del hábitat (Feng et al. 2017). En este caso, dos géneros diferentes dominaron los agroecosistemas conformados con la misma planta hospedera, pero el tamaño del cultivo puede estar favoreciendo otra especie de parasitoide. Los géneros dominantes en los tres sitios, corresponden a las especies Eiphosoma viticolle, Microcharops anticarsiae y Pristomerus spinator, que han sido registradas como parasitoides de $S$. frugiperda en maíz (Yu et al. 2016). El otro género dominante en la selva fue Casinaria, de la subfamilia Campopleginae, este parasitoide tiene rangos estrechos de hospederos y ataca hospederos expuestos (Townes 1969), lo que sugiere que en la selva puede 


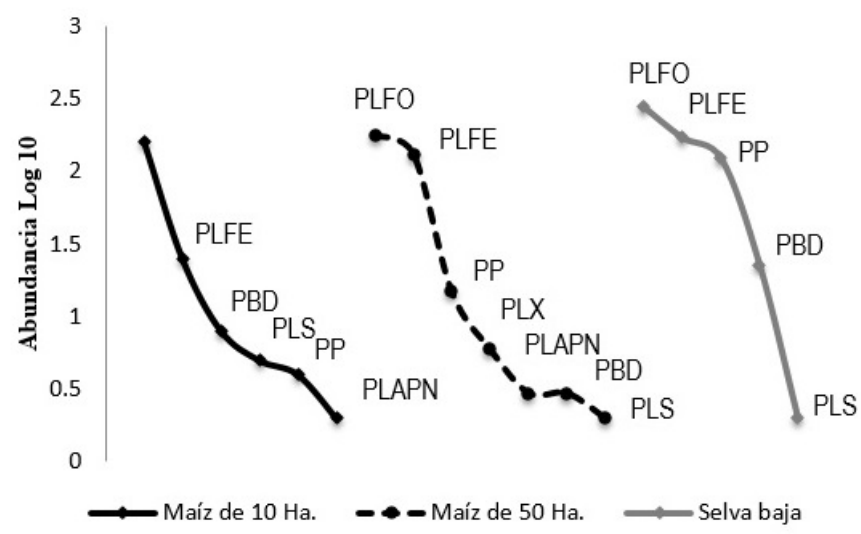

Figura 3. Curvas de Whittaker de gremios de Ichneumonidae en sitios con maíz de 10 y 50 ha y una selva baja caducifolia circundante. Los gremios son PLFO, parasitoides de larvas fitófagas ocultas; PLFE, parasitoides de larvas fitófagas expuestas; PP, parasitoides de pupas; PBD, parasitoides con biología desconocida; PLX, parasitoides de larvas xilófagas; PLS, parasitoides de larvas saprófagas; PLAPN parasitoides de larvas que se alimentan de polen y nectar.

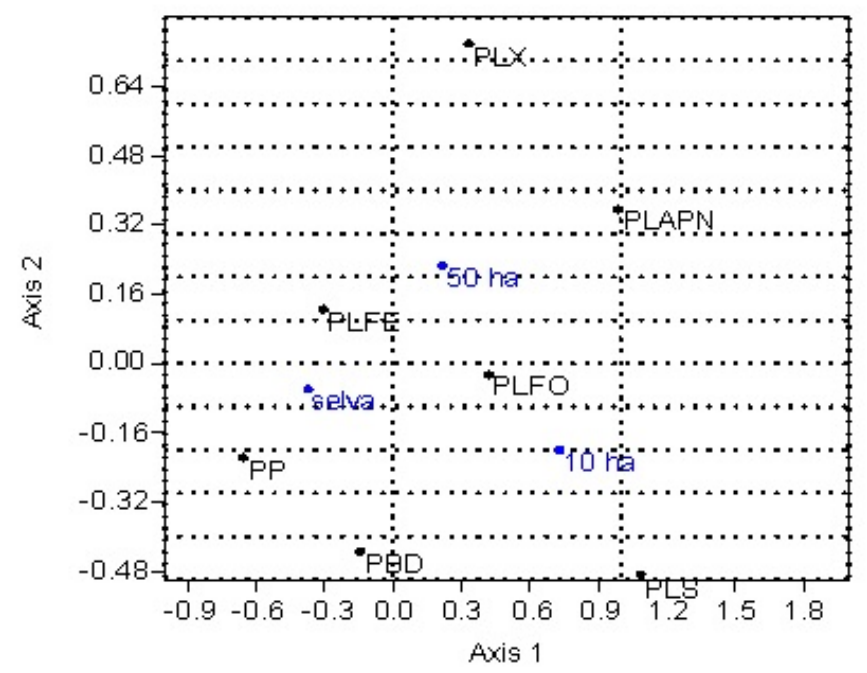

Figura 4. Análisis de correspondencia para gremios tróficos de Ichneumonidae para tres sitios, una plantación de maíz de 10 y 50 ha y la selva baja caducifolia circundante. Axis 1, Eigenvalue: 0.181939 (88.23\% del total); Axis 2, Eigenvalue: 0.0242639 (11.77\% del total). Los gremios son PLFO, parasitoides de larvas fitófagas ocultas; PLFE, parasitoides de larvas fitófagas expuestas; PP, parasitoides de pupas; PBD, parasitoides con biología desconocida; PLX, parasitoides de larvas xilófagas; PLS, parasitoides de larvas saprófagas; PLAPN parasitoides de larvas que se alimentan de polen y nectar.

estar presente en mayor abundancia los hospederos de este género. En México, Pristomerus es uno de los géneros considerados de mayor importancia en cuanto a distribución y porcentaje de para- sitismo de S. frugiperda (García-Gutierrez et al. 2013, Coronado-Blanco et al. 2017).

El tamaño del cultivo tiene un papel importante en la diversidad de los parasitoides, ya que pese a 
ser un monocultivo, el cultivo de maíz de 50 ha alberga la misma diversidad que la selva circundante. Esto puede deberse a que las selvas tienen estructuras complejas de vegetación (Hernández-Stefanoni et al. 2011), un mayor número de especies de árboles y herbáceas, así como mayor número de especies de hoja ancha (Fraser et al. 2007), lo que permite alojar mayor diversidad de insectos herbívoros y por consiguiente de parasitoides (Sääksjarvi et al. 2006). Probablemente la diversidad similar en la selva y el cultivo de maíz de 50 ha se deba a razones diferentes, ya en que la selva puede estar relacionada con la diversidad vegetal, y en el cultivo de maíz se deba a los volátiles inducidos por diferentes herbívoros que atacan el maíz o que al ser un área de mayor tamaño, este ofreciendo mayor número de refugios disponibles en comparación con el cultivo de maíz de 10 ha, lo que se refleja en mayor diversidad.

Los gremios más abundantes, coinciden con lo registrado en otros estudios (Mazón y Bordera 2014, González-Moreno et al. 2018), ya que se estima que hay de 5 a 6 especies de parasitoides por cada insecto fitófago (Hawkins y Lawton 1987). Además, la abundancia de los gremios PLFO, son los que se esperaban encontrar dada su biología de parasitoides de hospederos ocultos, ya que en ambos sitios con cultivo de maíz se encontraron larvas de su principal fitófago, el gusano cogollero ( $S$. frugiperda), que ataca las partes tiernas del maíz o cogollo. Las diferencias en la diversidad de gremios de los tres sitios, se relaciona con el tamaño del parche, lo que refuerza la teoría del tamaño del área propuesta por la biogeografía de islas (MacArthur y Wilson 1967). El encontrar un tercer gremio dominante en la selva, que no fue dominante en los cultivos de maíz, puede deberse a las estrategias de búsqueda de estos parasitoides generalistas; en la selva hay mayor disponibilidad de hospederos potenciales y no hospederos, que favorecen la presencia de parasitoides con hábitos más generalistas, porque son más eficientes para encontrar a sus hospederos en estos ambientes heterogeneos. Además, en la selva no se encontraron los gremios PLX y PLAPN, que si estuvieron presentes en los cultivos de maíz. En otros estudios los parasitoides PLX han mostrado una preferencia por madera de plantas arbustivas y no por madera de árboles viejos (Idris 2003), como podría tener en mayor proporción la selva en comparación con los agroecosistemas de maíz. También los parasitoides PLAPN que atacan larvas que se alimentan de polen y néctar, es probable que puedan ser favorecidos en la búsqueda de sus hospederos, en ambientes más homogeneos como el cultivo de máiz. El encontrar mayor número de gremios en el agroecosistema maíz, sugierie que los agroecosistemas pueden tener su propia diversidad, con varios nichos que albergan diferentes gremios de parasitoides, pese a ser un sistema simplificado en comparación con la heterogeneidad de la selva.

\section{CONCLUSIONES}

La diversidad de parasitoides fue mayor en el cultivo de maíz de mayor superficie, albergando comunidades de parasitoides tan diversas y similares como las que se encuentran en sistemas naturales. La composición de las comunidades en sitios con cultivo de maíz y selva fueron similares, aunque cada comunidad estuvo dominada por géneros diferentes de parasitoides característicos de las particularidades que podría presentar cada hábitat. Lo que refleja la importancia de tener alrededor de los cultivos remanentes de vegetación natural, que permita mantener altos valores de diversidad de parasitoides.

\section{AGRADECIMIENTOS}

Al Ingeniero Roberto Novelo por facilitar las parcelas para el establecimiento de los sitios de muestreo y al M. en C. Walther Torres Cab por colaborar en los trabajos de campo. Este trabajo estuvo financiado por el proyecto del Tecnológico Nacional de México (Referencia: 6255.17-P), titulado "Efecto de la superficie del cultivo de maíz sobre la diversidad y emergencia de parasitoides". 


\section{LITERATURA CITADA}

Chay-Hernández DA, Delfín-González H, Parra-Tabla V (2006) Ichneumonoidea (Hymenoptera) community diversity in an agricultural deciduas del Trópico: Una discusión acerca de su posible uso. Acta Zoológica Mexicana 79: 43-56.

Colwell (2016) EstimateS: Statistical Estimation of Species Richness and Shared Species from Samples, Version 9.10. http://viceroy.eeb.uconn.edu/estimates/. Fecha de consulta 6 de enero de 2019.

Coronado-Blanco J, Ruíz-Cancino E, Reséndiz-Ramírez Z, Estrada-Virgen O, Cambero-Campos O (2017) Ichneumonoidea (Hymenoptera) colectados en maíz en Localidades de Jalisco, Nayarit y Tamaulipas, México. Entomología Mexicana 4: 759-764

Delfín-González H, Bojórquez-Acevedo M, Manrique-Saide P (2007) Parasitoids of fall armyworm (Lepidoptera: Noctuidae) from a traditional maize crop in the Mexican State of Yucatan. Florida Entomologist 90: 759-761.

Feng Y, Kravchuk O, Sandhu H, Wratten S, Keller M (2017) The activities of generalist parasitoids can be segregated between crop and adjacent non-crop habitats. Journal of Pest Science 90: 275-286

Fraser SEM, Dytham C, Mayhew PJ (2007) Determinants of parasitoid abundance and diversity in woodland habitats. Journal of Applied Ecology 44: 352-361.

Gauld ID (1991) The Ichneumonidae of Costa Rica, 1. Memoirs of the American Entomological Institute 47: $1-589$.

Gauld ID (1997) The Ichneumonidae of Costa Rica, 2. Memoirs of the American Entomological Institute 57: $1-485$.

Gauld ID (2000) The Ichneumonidae of Costa Rica, 3. Memoirs of the American Entomological Institute 63: $1-453$.

Gauld ID (2002) The Ichneumonidae of Costa Rica, 4. Memoirs of the American Entomological Institute 66: $1-768$.

García-Gutiérrez C, González-Maldonado MB, González-Hernández A (2013) Parasitismo natural de Braconidae e Ichneumonidae (Hymenoptera) sobre Spodoptera frugiperda (Lepidoptera: Noctuidae). Revista Colombiana de Entomología 39: 211-215.

García-Ramírez M, Ruíz-Cancino E, Coronado-Blanco JM, Khalaim Al (2016) Ichneumonidae (Hymenoptera: Ichneumonoidea) de Escárcega, Campeche, México: nuevos registros de especies. Ciencia Universidad Autónoma de Tamaulipas 10: 06-12.

Garbarczyk H, Sawoniewicz J (1984) Classification of parasitoids into guilds associated with different host groups. Ekologia polska 32: 261-270.

Godfray HCJ (1994) Parasitoids. Behavioral and evolutionary ecology. Princeton University Press, Princeton, New Jersey, USA. 473p.

González-Maldonado MB, García-Gutiérrez C, González-Hernández A (2014) Parasitismo y distribución de Campoletis sonorensis Cameron (Hymenoptera: Ichneumonidae) y Chelonus insularis Cresson (Hymenoptera: Braconidae), parasitoides del gusano cogollero en maíz en Durango, México. Vedalia 15: 47-53.

González-Moreno A, Bordera S, Delfín-González H (2015) Spatio-temporal diversity of Cryptinae (Hymenoptera, Ichneumonidae) assemblages in a protected area of southeast Mexico. Journal of Insect Conservation 19: 1153-1161. 
González-Moreno A, Bordera S, Leirana-Alcocer J, Delfín-González H, Ballina-Gómez HS (2018) Explaining variations in the diversity of parasitoid assemblages in a biosphere reserve of Mexico: evidence from vegetation, land management and seasonality. Bulletin of Entomological Research 108: 602-615.

Gutiérrez-Ramírez A, Robles-Bermúdez A, Cambero-Campos J, Santillán-Ortega C, Ortíz-Catón M, CoronadoBlanco JM, Campos-Figueroa M (2015) Parasitoides de Spodoptera frugiperda (Lepidoptera: Noctuidae) Encontrados en Nayarit, México. Southwestern Entomologist 40: 555-564.

Hammer O, Harper DAT, Ryan PD (2001) PAST. Paleontological Statistics Sowftware Package for Education and Data Analysis. Paleontologia electronica 4: 1-9.

Hawkins BA, Lawton JH (1987) Species richness for parasitoids of British phytophagous insects. Nature 326 : 788-790.

Hay-Roe MM, Meagher RL, Nagoshi RN, Newman Y (2016) Distributional patterns of fall armyworm parasitoids in a corn field and a pasture field in Florida. Biological Control 96: 48-56.

Hernández-Stefanoni JL, Dupuy JM, Tun-Dzul F, May-Pat F (2011) Influence of landscape structure and stand age on species density and biomass of a tropical dry forest across spatial scales. Landscape Ecology 26: 355-370.

Hochberg ME, Ives AR (2000) Parasitoid population biology. Princeton University Press. USA. 366p.

Idris AB, Hanidah J, Gonzaga AD Azura N (2003) Diversity, abundance, species composition and similarity of genus Xanthopimpla (Ichneumonidae: Pimplinae) in logged and fragmented forests of the Langat basin in Selangor, Malaysia. Journal of Asia-Pacific Entomology 6: 55-62.

Inclán DJ, Cerretti P, Marini L (2014) Interactive effects of area and connectivity on the diversity of tachinid parasitoids in highly fragmented landscapes. Landscape Ecology 29: 879-889.

Landis DA, Wratten SD, Gurr GM (2000) Habitat management to conserve natural enemies of arthropod pests in agriculture. Annual Review of Entomology 45: 175-201.

MacArthur RH, Wilson EO (1967) The theory of island biogeography. Princeton University Press. Princeton, USA. 216p.

Maciel GAA, Araiza MDS (2015) Enemigos naturales de Spodoptera frugiperda y Helicoverpa zea como una alternativa al uso de insecticidas. Jóvenes en la Ciencia 1: 48-53.

Magurran AE (2004) Measuring biological diversity. Blackwell Publishing. Reino Unido. 255p.

Mazón M, Bordera S (2014) Diversity of Ichneumonidae (Insecta: Hymenoptera) in a protected area of Central Spain: What are we protecting? Insect Conservation and Diversity 7: 435-452.

Meagher RL, Nuessly GS, Nagoshi RN, Hay-Roe MM (2016) Parasitoids attacking fall armyworm (Lepidoptera: Noctuidae) in sweet corn habitats. Biological Control 95: 66-72.

Nuessly GS, Scully BT, Hentz MG, Beiriger R, Snook ME, Widstrom NW (2007) Resistance to Spodoptera frugiperda (Lepidoptera: Noctuidae) and Euxesta stigmatias (Diptera: Ulidiidae) in sweet corn derived from exogenous and endogenous genetic systems. Journal of Economic Entomology 100: 1887-1895.

Rojas BY, Ipagrraguirre CM, Rodríguez D (2017) Enemigos naturales en el cultivo de maíz en Ciego de Ávila. Universidad \& Ciencia 6: 27-41.

Ruíz-Cancino E, Rafaelevich-Kasparyan D, González-Moreno A, Ivanovich Khalaim A, Coronado-Blanco JM (2014) Biodiversidad de Ichneumonidae (Hymenoptera) en México. Revista Mexicana de Biodiversidad Supl. 85: S385-S391. 
Sääksjarvi IE, Ruokolainen K, Tuomisto H, Haataja S, Fine PVA, Cárdenas G, Mesones I, Vargas V (2006) Comparing composition and diversity of parasitoid wasps and plants in an Amazonian rain-forest mosaic. Journal of Tropical Ecology 22: 167-176.

SIAP (2018) http://www.siap.gob.mx/cierre-de-la-produccion-agricola-por-cultivo/. Fecha de consulta 6 de enero de 2019.

Townes $\mathrm{H}$ (1969) The genera of Ichneumonidae, Part 1. Memoirs of the American entomological Institute 11: $1-300$.

Valdez-Torres JB, Soto-Landeros F, Osuna-Enciso T, Báez-Sañudo A (2012) Modelos de predicción fenológica para maíz blanco (Zea mays I.) y gusano cogollero (Spodoptera frugiperda J. E. Smith). Agrociencia 46: 399-410.

Veijalainen A, Sääksjärvi IE, Erwin TL, Gómez IC, Longino JT (2013) Subfamily composition of Ichneumonidae (Hymenoptera) from western Amazonia: Insights into diversity of tropical parasitoid wasps. Composición de las subfamilias de Ichneumonidae (Hymenoptera) de la Amazonía occidental: Reflexiones sobre la diversidad de avispas parasitoides tropicales. Insect Conservation an Diversity 6: 28-37.

Yu DS, van Achterberg K, Horstmann K (2016) World Ichneumonoidea 2015. Taxonomy, biology, morphology and distribution. Taxapad. Vancouver, Canada. Flash drive. 
